\title{
Prediction of the Joint Toxicity of Multiple Engineered Nanoparticles: The Integration of Classic Mixture Models and In Silico Methods
}

Fan Zhang, Zhuang Wang, Martina G. Vijver, and Willie J. G. M. Peijnenburg*

Cite This: Chem. Res. Toxicol. 2021, 34, 176-178

Read Online

ACCESS | Lلll Metrics \& More 回 Article Recommendations

ABSTRACT: An approach to solve the emerging need of prediction of the toxicity of mixtures of engineered nanoparticles (ENPs) is presented. The integration of classic approaches to mixture toxicity assessment and computational toxicology approaches is proposed to be a smart strategy for forecasting the toxicity of a mixture of ENPs.

$\mathrm{V}$ arious nanostructured materials have been found to be attractive for applications due to their distinctive physicochemical properties from atoms, molecules, and traditional bulk materials. With the increasing market entry of existing nanomaterials and the uninterrupted development of new nanomaterials, a wide diversity of engineered nanoparticles (ENPs) will inevitably be released into the atmosphere, water, and soil, which also raises concerns about the risks of these ENPs to human and ecological health. ${ }^{1}$ Assessing the environmental risks of ENPs is not only a challenge for the scientific community but also a major international demand for the sustainable development of nanotechnology.

The presence of chemical contaminants in the form of mixtures in the environment is a common rule rather than an exception. Due to the extensive use of ENPs in industrial and consumer products, ENPs will inevitably be released into the environment. Natural ecosystems will thus be exposed to a mixture of ENPs. At present, with the ongoing development and application of nanocomposites, different types of ENPs will be jointly discharged into the environment. In addition, sewage systems and municipal wastewater treatment plants have become important in-between ways to transfer ENPs to the environment. Thereupon, industrial and municipal sewage is viewed as the main source of mixtures of ENPs of varying composition. The formulation of current environmental quality standards and risk assessments is usually based on toxicity data of individual ENPs, ${ }^{2}$ and the potential hazards of their combined toxic effects are not fully considered. This may be because the intrinsic toxicity of a mixture of ENPs (addition, synergism, or antagonism) is strikingly different from the toxicity of the individual ENPs. Meanwhile, a standardized and trustworthy method for estimation of ENP-mixture toxicity is lacking.

We propose that the key strategy to quantitatively predict the joint toxicity of an emerging or untested/unknown mixtures of multiple ENPs is to integrate the classical mixture toxicology methods with computational toxicology approaches.
This integration not only allows to properly incorporate the characteristics of the underlying mechanisms of toxicity (from the intrinsic structure information) as based on the classical mixture toxicology approaches but also strengthens the theorization (from the basic principles of mixture toxicology) of computational toxicology approaches.

On the basis of the classical mixture toxicology, the toxicity induced by exposure to mixtures of ENPs can be assessed using a component-based (CB) method. The CB method relies upon the response of the individual components of a mixture to predict the joint toxicity of the mixture. ${ }^{3}$ The $\mathrm{CB}$ method for the toxicity prediction of chemical mixtures can be further classified as following joint actions in toxicodynamic processes: similar joint action (concentration addition, CA), dissimilar joint action (independent action, IA), and synergistic/antagonistic actions. The CA and IA models are usually applied to quantify the joint toxicity (including toxicity indicators and full dose/concentration-response/effect relationships) of chemical mixtures. CA is accustomed to predicting the toxicity of mixtures consisting of chemicals with a similar mode of toxic action (MOA), and IA is conditioned to predicting the toxicity of mixtures comprising chemicals with a dissimilar MOA. Joint action as induced by chemical mixtures is a fundamental hypothesis. ${ }^{4}$ Note that the assessment of distinctive types of MOA of individual ENPinduced toxicity is prerequisite for the assessment of joint action of ENP mixtures. Furthermore, CA and IA have become two prevailing approaches for the quantitative prediction of mixture toxicity based upon the hypothesis of joint action. It is reasonable to believe that the widely accepted approaches to modeling mixture toxicity are applicable to mixtures of ENPs.

Special Issue: Computational Toxicology

Published: September 14, 2020

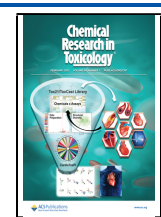




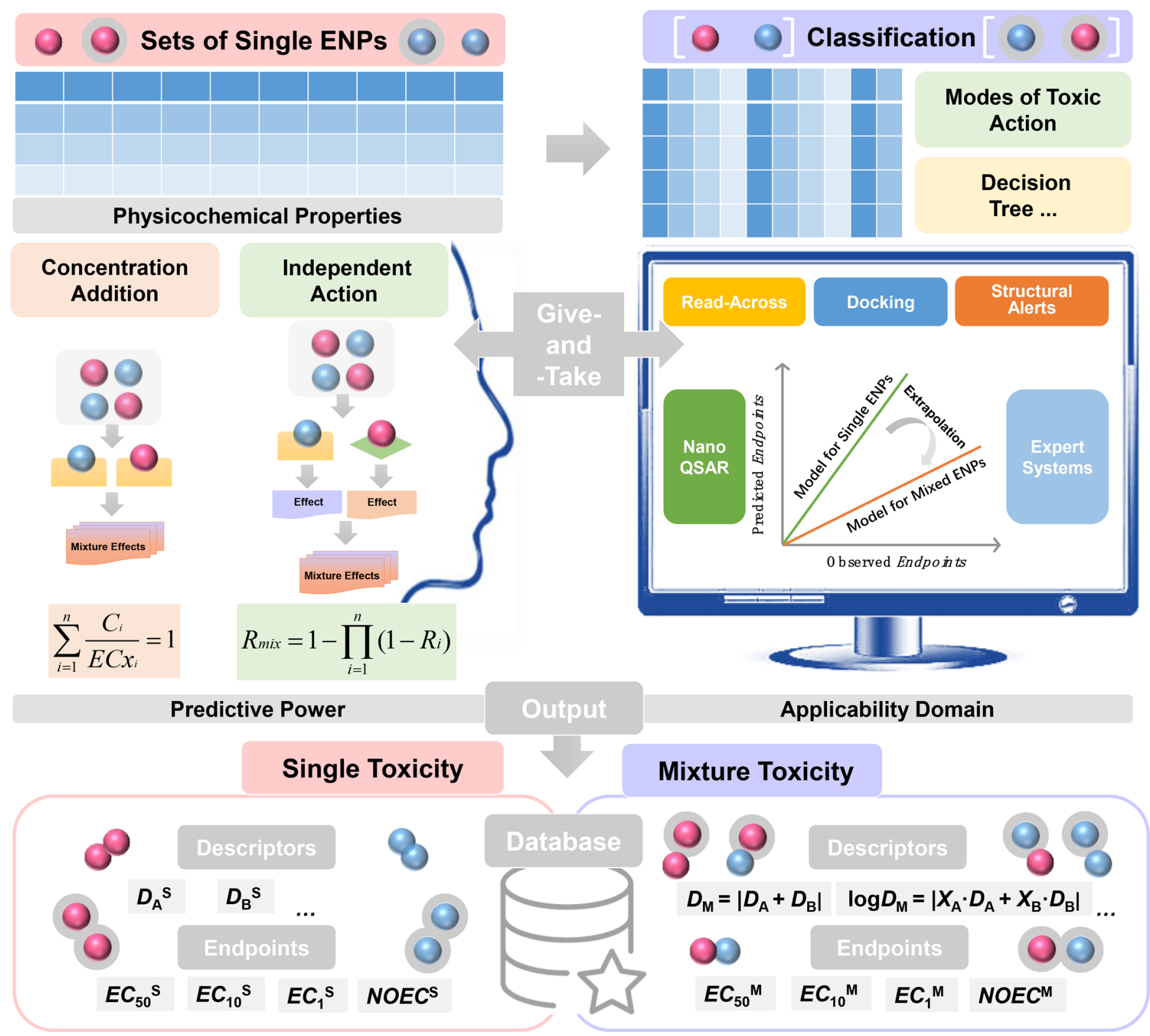

The development of nanotechnology has advanced by leaps and bounds, but our knowledge of the toxicological effects and risks of ENPs lags far behind the speed of their production and utilization. Most importantly, emerging nanomaterials are continuously being developed, introduced, and released. Nonetheless, different organism toxicity data are insufficient for single ENPs and yet are considerably scarcer for mixtures of ENPs. In addition, the experimental evaluation of the toxicity of single and mixtures of ENPs by means of biological models has a high cost and is time-consuming. In response to these issues, environmental scientists and toxicologists around the world have generally recognized the need to develop and validate theoretical prediction methods for ENPs, namely in silico (i.e., computational) toxicology models or predictivetoxicology models (mainly used for the prediction of toxicological indicators). ${ }^{5}$ Computational toxicology can offer powerful technique support to fill a vacancy in mixture toxicity data, as the majority of mixtures even lack toxicity data for the individual ENPs. Additionally, computational toxicology methods have the potential of being capable of in advance prediction of the joint toxicity of ENP mixtures of any composition. There have been notable examples of the prediction of toxicity of chemical mixtures through computational toxicology methods, ${ }^{4}$ for example, the quantitative structure-activity relationship (QSAR) model, the structural alerts model, the rule-based model, the uncertainty factors model and read-across, docking, and expert systems. Such methods could be applicable to the toxicity prediction of mixtures of ENPs.

The physicochemical and steric structures of ENPs determine the intrinsic mechanisms and linkages of their environmental behavior and toxicological effects, which are the core issues and challenges in constructing a predictive toxicology model, such as nano-QSAR. Through theoretical chemistry calculations such as molecular simulation, many nanodescriptors/parameters (such as energy levels, frontier orbital energy levels, charge, etc.) describing the characteristics of a single ENP structure can be obtained. Mechanism-based nanodescriptors for discriminating the toxicity of ENPs can be further screened via classification/regression methods, for example, decision tree, logistic regression, and support vector machine. The mechanism of toxicity of ENPs can also be 
revealed at the molecular level. The computation of descriptors for mixtures of ENPs is the real challenge. The weighted descriptor approach represents a preferred practice to work out descriptors for chemical mixtures. ${ }^{6}$

To achieve the integration of the classical mixture toxicity and computational toxicology methods, we suggest that research priorities for the prediction of the toxicity of mixtures of ENPs are identified to systematically sort out the toxicity and ecotoxicity information (macroscopic and microscopic toxicity end points) of ENPs gathered into "databases". Moreover, expected and/or actual environmental concentrations of ENPs need to be obtained to be implemented for actual risk profiling of various combinations of ENPs in the environment. These environmental concentrations can be further used for the estimation of mixture ratios of multiple ENPs, and then the weighted descriptors of ENP mixtures can be evaluated by the mixture ratios. Furthermore, it is essential that information on the MOA of single ENPs to species is systematically documented, and this issue deserves priority in the selection of methods to assess mixture toxicity and the selection of mechanism-based nanodescriptors.

\section{AUTHOR INFORMATION}

\section{Corresponding Author}

Willie J. G. M. Peijnenburg - Institute of Environmental Sciences (CML), Leiden University, Leiden 2300 RA, The Netherlands; Centre for Safety of Substances and Products, National Institute of Public Health and the Environment (RIVM), Bilthoven 3720 BA, The Netherlands; (1) orcid.org/ 0000-0003-2958-9149; Email: willie.peijnenburg@rivm.nl

\section{Authors}

Fan Zhang - Institute of Environmental Sciences (CML), Leiden

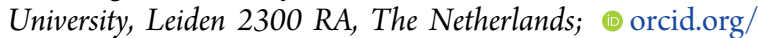
0000-0003-1709-7788

Zhuang Wang - School of Environmental Science and Engineering, Collaborative Innovation Center of Atmospheric Environment and Equipment Technology, Jiangsu Key Laboratory of Atmospheric Environment Monitoring and Pollution Control, Nanjing University of Information Science and Technology, Nanjing 210044, P.R. China; (1) orcid.org/ 0000-0001-7032-4500

Martina G. Vijver - Institute of Environmental Sciences (CML), Leiden University, Leiden 2300 RA, The Netherlands

Complete contact information is available at:

https://pubs.acs.org/10.1021/acs.chemrestox.0c00300

\section{Notes}

The authors declare no competing financial interest.

\section{ACKNOWLEDGMENTS}

The research described in this work was supported by the European Union's Horizon 2020 research and innovation program "NanoinformaTIX" (grant number 814426) and the National Natural Science Foundation of China (31971522). F.Z. greatly acknowledges the support from the China Scholarship Council (202008320308).

\section{REFERENCES}

(1) Peijnenburg, W. J. G. M., Baalousha, M., Chen, J., Chaudry, Q., Von Der Kammer, F., Kuhlbusch, T. A. J., Lead, J., Nickel, C., Quik, J. T. K., Renker, M., Wang, Z., and Koelmans, A. A. (2015) A review of the properties and processes determining the fate of engineered nanomaterials in the aquatic environment. Crit. Rev. Environ. Sci. Technol. 45, 2084-2134.

(2) Guinée, J. B., Heijungs, R., Vijver, M. G., and Peijnenburg, W. J. G. M (2017) Setting the stage for debating the roles of risk assessment and life-cycle assessment of engineered nanomaterials. Nat. Nanotechnol. 12, 727-733.

(3) Bopp, S. K., Barouki, R., Brack, W., Dalla Costa, S., Dorne, J. C. M., Drakvik, P. E., Faust, M., Karjalainen, T. K., Kephalopoulos, S., van Klaveren, J., Kolossa-Gehring, M., Kortenkamp, A., Lebret, E., Lettieri, T., Nørager, S., Rüegg, J., Tarazona, J. V., Trier, X., van de Water, B., van Gils, J., and Bergman, $\AA$ (2018) Current EU research activities on combined exposure to multiple chemicals. Environ. Int. 120, 544-562.

(4) Kar, S., and Leszczynski, J. (2019) Exploration of computational approaches to predict the toxicity of chemical mixtures. Toxics 7,15 .

(5) Chen, G., Peijnenburg, W. J. G. M., Xiao, Y., and Vijver, M. G. (2017) Current knowledge on the use of computational toxicology in hazard assessment of metallic engineered nanomaterials. Int. J. Mol. Sci. 18, 1504.

(6) Giner, B., Lafuente, C., Lapeña, D., Errazquin, D., and Lomba, L. (2020) QSAR study for predicting the ecotoxicity of NADES towards Aliivibrio fischeri. Exploring the use of mixing rules. Ecotoxicol. Environ. Saf. 191, 110004. 